\title{
Nicotinamide Riboside
}

\author{
National Cancer Institute
}

\section{Source}

National Cancer Institute. Nicotinamide Riboside. NCI Thesaurus. Code C158078.

An orally available form of vitamin B3 and precursor of nicotinamide adenine dinucleotide $(\mathrm{NAD+})$ with potential use in the treatment of chemotherapy induced peripheral neuropathy (CIPN). Upon oral administration, nicotinamide riboside (NR) is converted to nicotinamide mononucleotide by the NR kinases, nicotinamide riboside kinase 1 (NRK 1) and nicotinamide riboside kinase 2 (NRK 2), to which a second adenine is transferred by nicotinamide mononucleotide adenylyl transferase to generate NAD+. NAD+, an essential redox coenzyme, may offer protective effects against axonal injury from both mechanical and neurotoxic injury, and maintenance of NAD+ may be protective in mitochondrial disease. NR may help elevate and maintain NAD+ levels, which may ameliorate potential mechanisms implicated in the development of CIPN including mitochondrial dysfunction and peripheral nerve degeneration. 